\title{
Long-Term Outcomes of Patients Who Underwent Ureterocutaneostomy
}

\author{
(D) Fuat Kızılay MD, (D) Adnan Şimşir MD, (D) ibrahim Cüreklibatır MD, (D) Çağ Çal MD \\ Ege University Faculty of Medicine, Department of Urology, Izmir, Turkey
}

\begin{abstract}
Objective: Ureterocutaneostomy (UCS) is a urinary diversion (UD) method which is used rarely in a carefully selected patient group. In this study, we aimed to present the data and long-term outcomes of patients who underwent UCS treatment in our clinic.

Materials and Methods: A total of 36 patients who underwent UCS between January 2000 and December 2017 were included in the study. All patients had unilateral or bilateral ureteral-skin anastomosis. The demographic data, diagnoses, comorbidities, side of UCS (unilateral-bilateral), anesthesia method, operation time, and complications of the patients were recorded from the hospital registry system. The complications and survival status of the patients until the study date were evaluated. The study data were presented as mean (minimum-maximum) and number (percent).

Results: Mean follow-up time was 128.5 (8-192) months. The mean American Society of Anesthesiologists score was 3.25 . The patients had significant comorbidities, mainly hypertension, chronic obstructive pulmonary disease, and coronary artery disease. UCS was performed most frequently after radical cystectomy due to muscle-invasive bladder tumour (75\%) and was usually bilateral (75\%). The most common indications for UCS were significant comorbidities (55\%). Only 3 (8.33\%) patients developed ureteral-skin anastomosis, $6(16.66 \%)$ patients developed pyelonephritis, and 9 patients died during the follow-up period.

Conclusion: Although UCS is not a first-line UD method, it should still be kept in mind in the $21^{\text {st }}$ century for patients unable to tolerate segment excision from the gastrointestinal tract and postoperative complications due to comorbidities.
\end{abstract}

Keywords: Ureterocutaneostomy, urinary diversion, bladder tumour, survival, complication

\section{Introduction}

Ureterocutaneostomy (UCS) was first described by Johnston (1) 40 years ago as a bilateral end-to-end cutaneous ureterostomy for urinary diversion (UD). Johnston initially described it as a diversion method for children with congenital urinary obstruction, and it was later used in adults with pelvic malignancy.

Bladder tumors are the fourth most common tumor in men and the second most prevalent of all genitourinary system tumors. At time of diagnosis, $20-25 \%$ of tumors are muscle-invasive. The gold standard treatment of muscle-invasive tumors includes radical cystectomy, extended lymph node dissection, and UD
(2). Surgery for invasive bladder cancer may be conducted for curative or palliative purposes. If a palliative intervention is planned, the simplest and quickest method of diversion is best.

UD was first described by Simon (3) in 1852, and after the ileal conduit (IC) technique was popularized by Bricker (4) in 1950, this technique became a standard treatment method. UD is not practiced only after cystectomy, but is also used in cases of neurogenic bladder and congenital anomalies of the bladder. UD procedures performed for these reasons do not require cystectomy. The abdominal wall, urethra, and rectosigmoid colon are the main sites involved in UD (5). The patient's general health status, disease-specific health condition, expectations 
of postoperative quality of life, and renal function are factors evaluated when choosing UD type. Orthotopic ileal neobladder substitution seems to be the method that best complies with the physiological anatomy and provides the patient with the best outcomes in terms of quality of life and cosmesis. However, this method has some drawbacks. The main disadvantages of orthotopic substitution include the presence of a locally advanced bladder tumor and involvement of the urethra or bladder neck, long-term complication rates, the possible need for clean intermittent catheterization, and being a long and technically difficult surgical procedure. In such cases, incontinent UD types such as IC and UCS serve as alternative methods. As UCS does not involve taking a segment from the gastrointestinal system (GIS), patients are spared the potential adverse effects of this additional intervention. In particular, UCS is preferable as palliative treatment in cases with significant comorbidities, limited life expectancy, history of or plan for future intestinal radiotherapy (RT), or comorbid intestinal pathologies (ulcerative colitis, Crohn's disease) (6). In this study, we aimed to evaluate the long-term results of patients who underwent UCS in our clinic between 2000 and 2016.

\section{Materials and Methods}

\section{Patient Selection and Data Collection}

A total of 36 patients who underwent UCS between January 2000 and December 2017 were included in the study. Patients' demographic data, diagnoses, additional diseases, side of UCS (unilateral or bilateral), anesthesia method, operation time, and complications were recorded from the hospital records system. Signed informed consent forms were obtained from all patients for the UCS procedure. Postoperative complications and survival data until the date of the study were recorded. The study was designed in accordance with the Declaration of Helsinki and because the study was retrospective, ethics committee approval was not obtained.

\section{Ureterocutaneostomy Procedure}

Patients were operated in the supine position under general anesthesia or with a combination of epidural and spinal anesthesia. A median incision was made. The ureters were bilaterally (or unilaterally) dissected down to the ureterovesical junction and cut and spatulated after this point. A 4 Fr ureteral catheter was inserted into the ureter lumen and fixed with 2/0 Vicryl Rapide sutures from the middle of the ureter. The ureters were extruded from the skin lateral to the rectus muscle and fixed on the rectus abdominis fascia with $4 / 0$ Vicryl sutures at 12,3,6, and 9 o'clock approximately $3.5 \mathrm{~cm}$ proximally from the endpoint. The distal end of the ureter was also fixed to the skin with 4/0 Vicryl sutures from four quadrants. Immediately after the procedure, the UCS orifices were taken into the stoma in the operation room. The ureteral catheters were removed on postoperative day 10.

The study data were expressed as means (minimum-maximum) and numbers (percentage).

\section{Results}

The mean age of the patients was 74.4 years and $66.7 \%$ were male. The mean follow-up period was 128.5 months (range 8-192 months). Patients were at moderate to high risk regarding anesthesia, with a mean American Society of Anesthesiologists (ASA) score of 3.25 (range 2-5). Surgery was conducted under a combination of epidural and spinal anesthesia in 24 patients (66.6\%) and under general anesthesia in 12 patients (33.4\%). The mean surgery time was 89.4 minutes. UCS usually followed radical cystectomy performed due to muscle-invasive bladder tumor $(75 \%)$. UCS indication was based on the presence of significant comorbidities in 20 patients $(55.5 \%)$, history of pelvic RT in 12 patients (33.3\%), and presence of inflammatory bowel disease in 4 patients (11.1\%). In our patients, the UCS procedure was usually bilateral $(75 \%)$. Six of the 9 patients who underwent unilateral UCS had solitary kidneys and these patients were over the age of 70 years and had multiple comorbidities. The demographic data of the patients and the characteristics of the UCS procedure are summarized in Table 1. During follow-up, only 3 (8.33\%) patients developed ureterocutaneous anastomotic stricture at postoperative 98 months, which was treated by ureteral dilation and implantation of a 3-month $6 \mathrm{Fr}$ double-J stent. Pyelonephritis developed in 6 patients and 9 patients died during follow-up. The causes of mortality were systemic metastases due to bladder tumor recurrence in 5 patients and myocardial infarction in 4 patients. The patients' long-term follow-up data and complications are shown in Table 2.

\begin{tabular}{|l|l|}
\hline $\begin{array}{l}\text { Table 1. Demographic characteristics of ureterocutaneostomy } \\
\text { patients }\end{array}$ \\
\hline Variable & Value* \\
\hline Patient number & 36 \\
\hline Age (years) & $74.4(54-81)$ \\
\hline BMI (kg/m²) & $23.8(21.2-27.5)$ \\
\hline Sex & \\
Female & $12(33.3)$ \\
Male & $24(66.7)$ \\
\hline Comorbid diseases & \\
Diabetes mellitus & 15 \\
Hypertension & 27 \\
Chronic obstructive pulmonary disease & 24 \\
Coronary artery disease & 24 \\
Chronic kidney disease & 14 \\
Crohn's disease & 12 \\
\hline UCS indications & \\
Major comorbidities & $20(55.5)$ \\
Pelvic RT history & $12(33.3)$ \\
Inflammatory bowel disease & $4(11.1)$ \\
\hline ASA score & $3.25(2-5)$ \\
\hline Anesthesia method & $12(33.4 \%)$ \\
General anesthesia & $24(66.6 \%)$ \\
Spinal + epidural anesthesia & $27(75)$ \\
\hline $\begin{array}{l}\text { Pathology with UCS (\%) } \\
\text { Muscle-invasive bladder tumor }\end{array}$ & $9(25)$ \\
Neurogenic bladder & $9(25)$ \\
\hline UCS procedure & $27(75)$ \\
Unilateral & $89.4(30-150)$ \\
Bilateral & American Society of \\
\hline Surgery duration (min) & \\
\hline BMI: Body mass index, RT: Radiotherapy, ASA: \\
Anesthesiologists, UCS: Ureterocutanostomy \\
*Values expressed as mean (minimum-maximum) or number (percent) \\
\hline
\end{tabular}




\begin{tabular}{|l|l|l|}
\hline \multicolumn{3}{|l|}{ Table 2. The ureterocutaneostomy patients' long-term follow-up data } \\
\hline Complications & Value* & Duration** \\
\hline $\begin{array}{l}\text { Ureterocutaneous } \\
\text { anastomosis stricture }\end{array}$ & $3(8.33)$ & $98(76-112)$ \\
\hline Pyelonephritis & $6(16.66)$ & $63.5(58-69)$ \\
\hline Ureteral retraction & $1(2.77)$ & 110.4 \\
\hline Stoma necrosis & $1(2.77)$ & 122.8 \\
\hline Death & $9(25)$ & $76.33(68-82)$ \\
\hline $\begin{array}{l}\text { *Values expressed as number (percent) } \\
\text { **Times are given as mean (minimum-maximum) in months }\end{array}$ \\
\hline
\end{tabular}

\section{Discussion}

Many factors are considered when selecting a method of UD, including the patient's age, manual dexterity, physical build, mental and physical condition, renal functions, any comorbid intestinal pathologies, and prognosis of the primary disease, as well as the patient's expectations, preferences, and fears, and the training, experience, and skill of the physician. In addition, the cost of the procedure must also be taken into account (7). As there is no single option suitable for every patient, it is important to know and consider the different diversion methods. The implantation of ureters to the skin (ureterocutaneostomy) is the easiest method of UD and is the only diversion method that does not require removing a segment from the GIS. The main indications for UCS are the planning of palliative treatment, the presence of significant comorbidities, low life expectancy, a history of intestinal RT or planned intestinal RT, and the presence of pathologies that preclude the use of an intestinal segment (ulcerative colitis, Crohn's disease) (6). There are high complication rates, especially involving stoma necrosis, stenosis, and stricture, and patients often require ureteral catheterization. Therefore, the IC is the most preferred method of UD (33$63 \%)(8,9,10)$. Formed by the anastomosis of the ureter to the skin, UCS is indicated for palliative purposes in patients with a poor general condition and significant comorbidities, and is selected for $1-10 \%$ of patients. When a UCS is formed, diversions through a single stoma connecting to a single bag can be created. This is achieved with transuretero-ureterostomy by end-to-side anastomosis of the thin ureter to the other ureter and UCS of this single ureter. Another option is to join the medial edges of the ureter ends and pass them through an opening in the anterior abdominal wall to create a single stoma with a double-end appearance. In this study, we analyzed the long-term results of patients for whom we preferred the UCS method.

Patient age is important when determining the diversion method. Particularly at advanced ages, compromised intestinal integrity and segment excision from the GIS result in impaired intestinal absorption and secretion, and these patients become more susceptible to metabolic disorders (11). In a study by Kozacıoğlu et al. (12) presenting the data of 27 patients who underwent UCS, the patients' ages ranged from 58 to 78 years. Similarly, in our study, the age range was between 54 and 81 years. It is known that age is among the main factors determining quality of life of patients after UD (13). However, conflicting results have been reported on the relationship between age and the quality of life expected after cystectomy. Saika et al. (14) compared health-related quality of life after radical cystectomy in patients aged 75 years and older who underwent IC, UCS, and orthotopic UD (OUD) procedures. They determined that there were no significant differences in the quality of life questionnaire results between the three groups and concluded that OUD is a method that can be utilized in elderly patients (14). We did not use a questionnaire that assessed the quality of life of our patients, but we did not encounter any substantial complaints regarding quality of life after UCS. Limitations in the social activities of our patients due to their comorbidities and advanced age may be an important factor in this.

UCS is a technique that is often preferred for patients with significant comorbidities. In diversion types that involve taking a segment from the GIS, it is expected that comorbidities would increase the risk of complications (15). Furthermore, the presence of serious comorbidities is one of the accepted indications for UCS (6). UD method should be discussed in detail with each patient and factors influencing UD selection, including the patient's preference, age, comorbidities, and the oncological characteristics of the tumor, should be considered. Being relatively easy to perform and having a low risk of complications makes UCS a first-line choice, especially for patients with multiple comorbidities. Kozacıoğlu et al. (12) reported that important comorbidities such as hypertension, chronic obstructive pulmonary disease, and diabetes were common among the patients in their study. We found that UCS was most commonly performed on high-risk patients with major comorbidities, and for this reason the operation was done under a combination of spinal and epidural anesthesia in $66.6 \%$ of patients.

The ASA score is a scoring system established by the ASA to predict patients' operative risk. It has been shown that UCS, the simplest incontinent diversion method, can reduce the risk of postoperative ileal and pulmonary complications, especially in patients with an ASA score of 3 or higher (16). Our patients had a mean ASA score of 3.25 and were at high anesthesia risk. For this reason, regional anesthesia was preferred for a large proportion of our patients. Longo et al. (17) compared the perioperative and quality of life outcomes of UCS and IC in patients over 75 years of age with ASA scores greater than 2 who underwent radical cystectomy, and reached the conclusion that UCS is a valid alternative that reduces perioperative complications and does not significantly affect quality of life in patients with significant comorbidities. Although the ASA scores of our patients were high, this method was considered relatively safer due to the short operation time, the use of regional anesthesia in most patients, and our desire to avoid the morbidity caused by alternative treatment methods such as RT and chemotherapy.

Prior or planned RT is a major obstacle to the use of the rectum segment for diversion, and may also warrant a UCS indication. One of the underlying causes of poor functional outcomes in elderly patients who undergo continent UD is a history of RT.

UD is not only performed for bladder malignancies or as a postcystectomy procedure, but can also be done for neurogenic 
bladder and congenital bladder anomalies. However, UCS is often performed in cases where intraoperative survival may be affected because of complications associated with locally advanced bladder tumor and intestinal and general health problems. OUD is not suitable in cases where the bladder tumor involves the bladder neck or for patients with renal dysfunction (creatinine clearance $<50 \mathrm{mg} / \mathrm{dL}$ ) and heart failure (ejection fraction $<45 \%$ ), and UCS or IC is often preferred for these patients (18). The incidence of bladder tumor is increasing across the world, leading to more radical cystectomy and UD procedures. UCS was performed for various reasons on our patients, but the most common reason was for UD after radical cystectomy due to muscle-invasive bladder tumor. When all stages of bladder tumor are taken into account, the 5-year survival rate is around $77 \%$ and the 10 -year survival rate is around $70 \%$. The 5-year survival rate falls to approximately $47 \%$ for localized muscle-invasive bladder tumors, which account for $35 \%$ of cases (19). In our patients, the 10 -year survival rate was $75 \%$. The main reasons for this were that the operation was performed for reasons unrelated to cancer in $25 \%$ of patients, and in those patients operated due to tumors, the procedure was performed within 90 days, which is a critical period for these patients.

In patients with solitary kidneys who have comorbidities and are in poor general condition, UCS may be a suitable method in order to avoid subjecting the patient to additional bowel surgery. The UCS procedure was performed bilaterally in the majority of our patients, but unilateral UCS was preferred in 9 patients with multiple comorbidities and poor general condition. The operation time can also be an important factor contributing to complication rates in these patients. The mean operation time was 89.4 minutes in our study.

Continent diversion methods offer certain advantages in terms of function and quality of life, but they are technically difficult to perform and the operation time is long, both of which contribute to high complication rates. They are also associated with high rates of reoperation, which may be needed in the long term. Compared to these procedures, UCS is technically easier to perform and is especially safer for patients with high anesthesia risk, and has lower complication rates. However, stoma problems are an important issue with UCS, and therefore, the IC method introduced in 1952 has largely replaced UCS. Nevertheless, UCS seems to cause less morbidity than IC. Kilciler et al. (20) reported that UCS was associated with shorter hospitalization time, less perioperative blood loss, and lower late complication rate compared to IC. However, another study showed that the type of diversion did not affect length of hospital stay (21). Furthermore, with the use of new stents, stoma problems have mostly been overcome. Some clinics attempt to prevent stomal obstruction by inserting a mono-j catheter into the ureter and replacing it every 2-3 months using a guidewire. However, serious stomal problems may occur despite this, and may require stomal reconstruction with open surgery. In a study analyzing 16 months of followup for incontinent diversion methods, UCS was found to be more advantageous than ileal and colonic conduits (22). There is consensus in the literature that UD types cannot be recommended randomly to patients and that of all types of
UD, UCS has the lowest surgical complication rate. Two large reviews emphasized that there is no scientific evidence that any diversion type is superior to others in terms of quality of life $(23,24)$. However, there is a need for large-scale, randomized, prospective studies in this area. Ureterocutaneous anastomotic stricture and pyelonephritis attacks were two major surgeryrelated complications in our patients, but these occurred at low rates $(8.33 \%$ and $16.66 \%$, respectively). Although different techniques have been modified, high rates of stomal stenosis have been reported in the literature (25). Toyoda (26) emphasized that with the technique they developed, the high rate of stenosis can be overcome by spatulating the ureter and implanting the stoma to skin separated from the epidermis and dermis. Kim et al. (27) reduced stenosis rates with a modified version of this technique in which they fixed the tunnel inside the abdominal wall between the anterior and posterior sheaths of the rectus fascia, and suggested that the main cause of stenosis is compression of the ureter by the tunnel in the abdominal wall. Our technique was similar to both of these methods, and we believe that ureteral spatulation and fixation of the tunnel to the abdominal wall contributed to our low rate of stomal stenosis. In addition, it has been speculated that obesity may contribute to stomal stenosis (28) and the mean body mass index of our patient group was 23.8 (normal weight). We detected stomal necrosis in one patient at postoperative 122 months. This patient was a 78-year-old man with uncontrolled diabetes and severe peripheral vascular disease. In this patient, ischemia at the microvascular level due to his comorbidities may have led to the development of this destructive complication.

\section{Study Limitations}

The drawbacks of our study are its retrospective nature, the fact that the surgical procedures were performed by different surgeons, and the lack of a comparative control group. However, we believe that the long follow-up period and the large patient group are strengths of our study because of the limited data in the literature about UCS, which is a rarely performed type of UD.

\section{Conclusion}

UCS should not be considered as a first option for patients who will undergo UD. However, diversion with UCS should still be kept in mind in the $21^{\text {st }}$ century for selected patients with low life expectancy and significant comorbidities, those with a contraindication for obtaining a GIS segment, and those who cannot tolerate postoperative complications.

\section{Ethics}

Ethics Committee Approval: Because the study was retrospective, ethics committee approval was not obtained.

Informed Consent: It was obtained.

Peer-review: Externally peer-reviewed.

\section{Authorship Contributions}

Surgical and Medical Practices: F.K., A.Ş., İ.C., Ç.Ç. Concept: F.K., A.Ş., Design: F.K., A.Ş., Data Collection or Processing: F.K., 
A.Ş., Analysis or Interpretation: F.K., A.Ş., I.C., Ç.Ç., Literature Search: F.K., A.Ş., Writing: F.K., A.Ş.

Conflict of Interest: No conflict of interest was declared by the authors.

Financial Disclosure: The authors declared that this study received no financial support.

\section{References}

1. Johnston JH. Temporary cutaneous ureterostomy in the management of advanced congenital urinary obstruction. Arch Dis Child 1963;38:161-166.

2. Khosravi-Shahi P, Cabezón-Gutiérrez L. Selective organ preservation in muscle-invasive bladder cancer: Review of the literature. Surg Oncol 2012;21:17-22.

3. Simon J. Operation for directing the orifices of ureters into the rectum; temporary success, subsequent failure; autopsy. Lancet 1852:568-570.

4. Bricker EM. Bladder substitution after pelvic evisceration. Surg Clin North Am 1950;30:1511-1521.

5. Stenzl A, Cowan NC, De Santis M, et al. Treatment of muscle-invasive and metastatic bladder cancer: update of the EAU guidelines. Eur Urol 2011;59:1009-1018.

6. Yoshimura K, Maekawa S, Ichioka K, et al. Tubeless cutaneous ureterostomy: the Toyoda method revisited. JUrol 2001;165:785-788.

7. Hautmann RE, Volkmer BG, Schumacher MC, et al. Long-term results of standard procedures in urology: the ileal neobladder. World J Urol 2006;24:305-314

8. Somani BK, Nabi G, Wong $S$, et al. How close are we to knowing whether orthotopic bladder replacement surgery is the new gold standard?--evidence from a systematic review update. Urology 2009;74:1331-1339.

9. Lowrance $W T$, Rumohr JA, Clark PE, et al. Urinary diversion trends at a high volume, single American tertiary care center. J Urol 2009;182:2369-2375.

10. World Health Organization (WHO) Consensus Conference on Bladder Cancer1, Hautmann RE, Abol-Enein $\mathrm{H}$, et al. Urinary diversion. Urology 2007;69(1 Suppl):17-49.

11. Stein R, Schröder A, Thüroff JW. Bladder augmentation and urinary diversion in patients with neurogenic bladder: non-surgical considerations. J Pediatr Urol 2012;8:145-152.

12. Kozacıoğlu Z, Değirmenci T, Günlüsoy B, et al. Ureterocutaneostomy: for whom and when? Turk J Urol 2013;39:143-146.

13. Bossi A. Re: Arnulf Stenzl, Nigel C. Cowan, Maria De Santis, et al. Treatment of muscle-invasive and metastatic bladder cancer: update of the EAU Guidelines. Eur Urol 2011;59:1009-18. Eur Urol 2011;60:e53.

14. Saika T, Arata R, Tsushima T, et al. Health-related quality of life after radical cystectomy for bladder cancer in elderly patients with an ileal conduit, ureterocutaneostomy, or orthotopic urinary reservoir: a comparative questionnaire survey. Acta Med Okayama 2007;61:199-203.

15. McDougal WS. Metabolic complications of urinary intestinal diversion. J Urol 1992;147:1199-1208.

16. Lodde M, Pycha A, Palermo S, et al. Uretero-ureterocutaneostomy (wrapped by omentum). BJU Int 2005;95:371-373.

17. Longo N, Imbimbo C, Fusco F, et al. Complications and quality of life in elderly patients with several comorbidities undergoing cutaneous ureterostomy with single stoma or ileal conduit after radical cystectomy. BJU Int 2016;118:521-526.

18. Vakalopoulos I, Dimitriadis G, Anastasiadis A, et al. Does intubated uretero-ureterocutaneostomy provide better health-related quality of life than orthotopic neobladder in patients after radical cystectomy for invasive bladder cancer? Int Urol Nephrol 2011;43:743-748.

19. Howlader N, Noone A, Krapcho M, et al. SEER cancer statistics review, 1975-2009 (vintage 2009 populations), National Cancer Institute. Bethesda, MD. MD, USA 2012.

20. Kilciler M, Bedir S, Erdemir F, et al. Comparison of ileal conduit and transureteroureterostomy with ureterocutaneostomy urinary diversion. Urol Int 2006;77:245-250.

21. Chang SS, Baumgartner RG, Wells N, et al. Causes of increased hospital stay after radical cystectomy in a clinical pathway setting. I Urol 2002;167:208-211.

22. Pycha A, Comploj E, Martini T, et al. Comparison of complications in three incontinent urinary diversions. Eur Urol 2008;54:825-832.

23. Porter MP, Penson DF. Health related quality of life after radical cystectomy and urinary diversion for bladder cancer: a systematic review and critical analysis of the literature. J Urol 2005; 173:1318-1322.

24. Gerharz EW, Månsson $\AA$, Hunt $S$, et al. Quality of life after cystectomy and urinary diversion: an evidence based analysis. J Urol 2005; 174:1729-1736.

25. MacGregor PS, Montie JE, Straffon RA. Cutaneous ureterostomy as palliative diversion in adults with malignancy. Urology 1987;30:31-34.

26. Toyoda $Y$. A new technique for catheterless cutaneous ureterostomy. J Urol 1977; 117:276-278.

27. Kim CJ, Wakabayashi Y, Sakano Y, et al. Simple technique for improving tubeless cutaneous ureterostomy. Urology 2005;65:1221-1225.

28. Namiki T, Yanagi S. A new technique for bilateral single stoma loop cutaneous ureterostomy. J Urol 1995;154:361-363. 